P98 ACCURACY OF ROUTINE ANTIBIOTIC SUSCEPTIBILITY TESTING OF SPUTUM SAMPLES IN ADULT CYSTIC FIBROSIS (CF) PATIENTS COLONISED WITH PSEUDOMONAS AERUGINOSA (PSA)

doi:10.1136/thx.2010.150979.49

${ }^{1} \mathrm{~A}$ Ashish, ${ }^{2} \mathrm{~J}$ Fothergill, ${ }^{2} \mathrm{E}$ Mowat, ${ }^{1} \mathrm{~S}$ Huq, ${ }^{1} \mathrm{M}$ Harris, ${ }^{1} \mathrm{C}$ Winstanley, ${ }^{1} \mathrm{M}$ Walshaw. ${ }^{1}$ Liverpool Heart and Chest Hospital, Liverpool, UK; ${ }^{2}$ University of Liverpool, Liverpool, UK

Background In CF patients with chronic Psa infection, the role of conventional antibiotic susceptibility testing (antibiotic disc diffusion on sputum subculture morphotypes according to BSAC protocols) is controversial, but many centres still rely on these methods when selecting treatment. However, morphotypes may exhibit up to six antibiograms, and this may contribute to the variable clinical response often noted. To look at this further, we performed additional Psa susceptibility testing on 26 sputum samples from 10 chronically infected CF patients provided over a 15month period, and compared the results with those from the routine laboratory.

Methods For each sputum sample, 40 colonies proportionately representative of morphological subtypes (mean 2, range 1-4) on the Psa selective plates were cultured onto Columbia plates. From these, single colonies were mixed with sterile distilled water to attain a standard optical density (10 MacFarland units), and $10 \mu \mathrm{l}$ spread onto iso-sense plates and incubated overnight with tobramycin, meropenem, colomycin, ceftazidime, ciprofloxacin, and piperacillin/tazobactam antibiotic discs. Antibiotic sensitivity (break point $50 \%$ of isolates) was determined by the zone of inhibition as per standardised BSAC protocols. In total, 6240 analyses were performed.

Results Although there was $100 \%$ concordance with the number of morphological subtypes of Psa with the routine laboratory, on multiple antibiotic susceptibility testing in 260 cases $(25 \%)$ increased resistance was discovered. Overall, mean concordance between the routine diagnostic lab methodology and multiple antibiotic sensitivity testing was $70 \%$ (median $80 \%$, IOR 60-100). However, in $15 \%$ of cases concordance was $<50 \%$, suggesting that more detailed testing may have altered the choice of antibiotics used. Conclusion This study shows that routine microbiological methodology may under-represent antimicrobial resistance in Psa when patients are chronically infected. This may in part explain the clinical experience, and underlines the need for better microbiological techniques to aid the clinician in caring for these complex patients.

\section{P99 SOCIAL DEPRIVATION AND CLINICAL OUTCOMES IN ADULT CF PATIENTS}

doi:10.1136/thx.2010.150979.50

A Ashish, D Nazareth, A Tsoulkani, G Priona, M Harris, M Ledson, M Walshaw. Liverpool Heart and Chest Hospital, Liverpool, UK

Introduction Economic and social deprivation is associated with increased morbidity and mortality, and this may be particularly important in chronic disease states such as cystic fibrosis, where there is a high burden of care. Despite this, there have been no studies assessing its impact on adult patients with CF. Our large adult CF clinic takes patients from a wide catchment area, including some of the most deprived areas in the country: we wished to study the impact of this deprivation on the health of our CF patients.

Methods We used the postcode-based Index of Multiple Deprivation (IMD) which takes into account seven domains (including income, education, housing and health-related outcomes), to assign Lower Super Output Areas (LSOAs) in a range of 0-32 486 (where a lower
LSOA indicates increased deprivation) to adult patients attending our centre. LSOAs were then correlated with spirometry, BMI, clinic attendances, number and length of inpatient spells, treatment burden, diabetes, and ultimate outcome between 2004 and 2009 . Data were analysed using the $\chi^{2}$ test and bivariate correlations to calculate Pearson's coefficient, where appropriate.

Results Of 219 patients (mean age 27.8 years, range 17-65, 99 females), $113(57 \%)$ lived in the lowest $20 \%$ of LSOAs (compared to only $20 \%$ nationally). We found no correlation between IMD score and FEV1, BMI, hospital admissions, diabetes and death, but there was an inverse relationship $\left(r^{2}=-0.153, p<0.05\right)$ with the mean length of inpatient stay.

Conclusion Although many of our adult CF patients live in very deprived areas, this seems to have little impact on their health over a 5 -year period, but this is at the expense of more inpatient care, a surrogate marker for more intensive treatment. It is therefore likely that these patients will consume more healthcare resource, and this may need to be factored into any proposed national policy allotting funding towards this complex group of patients.

\section{P100 AN INVESTIGATION OF MUTATOR PSEUDOMONAS AERUGINOSA IN CHRONICALLY INFECTED CYSTIC FIBROSIS (CF) PATIENTS TREATED FOR PULMONARY EXACERBATION}

doi:10.1136/thx.2010.150987.1

T Gouliouris, C R Laughton, D M Pearce, V Athithan, J E Foweraker. Papworth Hospital, Microbiology Department, Papworth Everard, UK

Background Pseudomonas aeruginosa with increased mutation rates due to defective DNA repair are reported in $24-73 \%$ of chronically infected patients with bronchiectasis, with or without cystic fibrosis (CF). Mutators may enhance $P$ aeruginosa persistence in the lung by accumulating adaptive mutations including antibiotic resistance, and have been associated with worse lung function. In chronic infection the sputum can contain a heterogeneous population of $P$ aeruginosa with different phenotypes, including variations in colony morphology (morphotype) and antibiotic susceptibility. Most studies on mutator prevalence however only tested one or two isolates per sputum. Investigation of the clinical relevance of mutator strains relies on accurately assessing the prevalence of hypermutators. We therefore performed a detailed investigation of mutators in sputum before and after antibiotic treatment for acute exacerbation.

Methods Six patients with CF, chronically infected with $P$ aeruginosa, were tested before and after 2 weeks antibiotic treatment for exacerbation. Culture, antibiotic susceptibility testing and pulsedfield gel electrophoresis (PFGE) typing were performed using published methods. ${ }^{1}$ Mutation rates were measured for up to four colonies of each morphotype. As the original phenotypic method for detecting mutators is very laborious, ${ }^{2}$ we developed a method to screen pooled cultures using spiral plater quantitation.

Results $168 P$ aeruginosa isolates were tested (average 14 per sputum). Three patients had no mutators before or after antibiotics and three cultured mutators before and after antibiotics. One to four PFGE pulsotypes and two to six morphotypes were found in each sputum. Both mutators and non-mutators were present in a single sample with no association with morphotype or genotype. Mutators were more likely to be antibiotic resistant, but there was no obvious selection of mutators after 14 days antibiotics.

Conclusions With extensive testing, mutators were found in three of six patients with chronic $P$ aeruginosa infection. The phenotypic and genotypic diversity observed means that multiple colonies of each morphotype should be tested to reliably detect mutators. Our method variation allows more extensive testing and may assist future clinical studies. 